\title{
Ressonância Magnética Cardíaca no Surto de COVID-19: Essencial ou Acessória?
}

\section{Cardiac Magnetic Resonance in the COVID-19 Outbreak: Essential or Subsidiary?}

Tiago Teixeira ${ }^{1}$, Fernando Pinto ${ }^{1}$

\section{RESUMO}

A pandemia da COVID-19, originada pelo novo coronavírus SARS-CoV-2, tem criado elevada morbimortalidade. As doenças cardiovasculares condicionam o prognóstico da mesma, havendo cada vez mais provas de afeção miocárdica pelo vírus, mas sendo a fisiopatologia da mesma ainda desconhecida. A ressonância magnética cardíaca, com a sua capacidade de avaliação das características tecidulares, poderá, quando utilizada no contexto correto, dar importantes informações de diagnóstico retrospetivo, essenciais para a orientação de futuros casos graves de COVID-19.

PALAVRAS-CHAVE: COVID-19; Miocardite/diagnóstico por imagem; Ressonância Magnética

\section{ABSTRACT}

The COVID-19 pandemic, originated by the new coronavirus SARS-CoV-2, generated high morbidity and mortality. Cardiovascular diseases affect its prognosis, with more and more evidence of myocardial affection by the virus, despite the pathophysiology of it is still unknown. Cardiac magnetic resonance, with its ability to assess tissue characteristics, may, when used in the correct context, provide important information for retrospective diagnosis, essential for the orientation of future serious cases of COVID-19.

KEYWORDS: COVID-19; Magnetic Resonance Imaging; Myocarditis/diagnostic imaging 
A pandemia originada pelo novo coronavírus, SARS-CoV-2, apanhou o mundo de assalto no final de 2019, originada na província de Hubei na China, estendendo-se a mais de 215 países, e ultrapassando, no final de abril de 2020, os 3.25 milhões de infetados e 230 mil óbitos. ${ }^{1}$ Com uma elevada taxa de transmissão, o vírus origina uma doença denominada COVID-19, de apresentação maioritariamente respiratória nos seus hospedeiros, caracterizada por uma pneumonia intersticial bilateral, com consolidações subpleurais, muitas vezes necessitando de suporte ventilatório, e condicionando elevada taxa de letalidade (cerca de 15\% nos maiores de 70 anos em Portugal no início de maio de 2020).

A afeção respiratória é, no entanto, apenas uma parte da fisiopatologia deste vírus. $O$ vírus utiliza a enzima ACE 2 , do eixo renina-angiotensina-aldosterona, para entrada nas células, ${ }^{2}$ enzima essa abundante no epitélio alveolar pulmonar, razão da apresentação respiratória dominante. A enzima está também presente no corpo humano no miocárdico, células vasculares e trato gastrointestinal. Dessa forma, estes sistemas de órgãos podem também ser afetados diretamente pelo vírus, além duma possível afeção indireta em caso de disfunção múltipla de órgãos, que a COVID-19 pode manifestar numa terceira fase da infeção, por provável tempestade de citocinas (Fig. 1). ${ }^{3}$ Estas variações de manifestação não são apenas importantes por nos dificultar o diagnóstico nalguns casos com apresentação mais atípica, aumentando a probabilidade de contaminação quer de conviventes quer de profissionais de saúde não protegidos que interajam com estes doentes, mas também pelo facto de condicionarem desfavoravelmente a sobrevida dos mesmos.

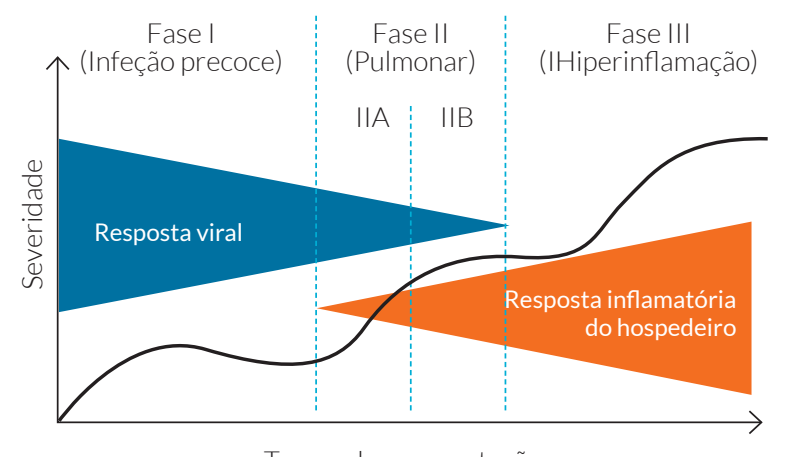

Tempo de apresentação

FIGURA 1. Observam-se as fases da COVID-19, uma primeira fase com agressão direta do vírus e uma terceira fase de resposta inflamatória do hospedeiro, passando por uma fase intermédia, habitualmente pulmonar.

Adaptado de Siddiai HK, et al. COVID-19 illness in native and immunosuppressed states: A clinical-therapeutic staging proposal. J Heart Lung Transplant. 2020;39:405-7.3
Na verdade, a doença cardiovascular condiciona de forma decisiva o prognóstico da COVID-19. Por um lado, os doentes mais gravemente afetados são aqueles com fatores de risco ou doenças cardiovasculares prévias, destacando-se a hipertensão arterial como o fator mais prevalente nos doentes com desfecho negativo (16,9\%). ${ }^{4}$ Estes doentes são habitualmente também os mais idosos, o fator de maior importância na letalidade da doença. ${ }^{5}$ Por outro lado, cada vez mais dados confirmam que a COVID-19 pode originar lesão miocárdica, com impacto no prognóstico. ${ }^{6}$ No entanto, o mecanismo fisiopatológico dessa afeção cardíaca não está ainda esclarecido, ${ }^{7}$ parecendo haver dois grandes perfis clínicos de afeção miocárdica, dependendo do período temporal em que a mesma ocorre. ${ }^{8}$ Um desses perfis, que denominaremos tardio, ocorre alguns dias após o início dos sintomas respiratórios, provavelmente englobado numa disfunção multiorgânica. De facto, a elevação dos marcadores bioquímicos de lesão e disfunção miocárdica, troponina I e peptídios natriuréticos respetivamente, nos indivíduos internados por apresentação respiratória, relacionou-se diretamente com um agravamento do prognóstico da doença, em alguns grupos de doentes chineses já reportados. ${ }^{9}$ O outro perfil poderá ser independente das manifestações respiratórias, ocorrendo desde o início da apresentação clínica (precoce). Noutra população que lidou mais precocemente com a doença, grupos italianos reportaram uma elevada proporção de ausência de doença coronária obstrutiva (60\%), entre os primeiros 33 doentes que se apresentavam como síndromes coronárias agudas (SCA) suspeitas e relativa ausência precoce de queixas respiratórias (dados não publicados). Estes indícios sugerem que o mecanismo de afeção cardiovascular precoce, ainda relativamente especulativo numa doença recente, possa ocorrer por um de dois mecanismos principais, a inflamação mediada pelo vírus condicionando miocardite, ou um desequilíbrio oferta-necessidade originando isquémia, sem exclusão da possibilidade de ocorrência de eventos vasculares agudos em consequência da elevada capacidade trombogénica, venosa e arterial, do vírus. Aliás, como ocorre frequentemente em medicina onde não existem fenómenos exclusivos, os diferentes mecanismos poderão ocorrer em diferentes indivíduos, mediante a suscetibilidade. Acresce ainda um outro fator de afeção cardíaca, este mais indireto, que é o medo da COVID-19. É que, além do salutar cumprimento de isolamento social imposto pelas autoridades que manteve muitos portugueses em casa durante várias semanas, este receio de contrair a doença nos hospitais, terá limitado que alguns doentes com SCA tenham recorrido à urgência hospitalar. Esta observação prende-se com uma redução de 
41\% no registo de SCA portugueses na primeira quinzena de março de 2020, altura em que o primeiro caso de infeção foi reportado, em relação ao período homólogo do ano anterior. Mesmo que se admita que a incidência de SCAs se tenha reduzido durante esta situação de exceção, sendo a limitação do stress do dia-a-dia e da poluição atmosférica alguns dos fatores postulados como responsáveis por essa eventual redução, alguns outros doentes ter-se-ão escusado de procurar o auxílio necessário, e, ou faleceram de morte súbita, ou irão procurar em breve as consultas ambulatórias de Cardiologia por queixas de insuficiência cardíaca ou angor residual.

A ressonância magnética cardíaca (RMC) tem-se afirmado nas últimas décadas como um dos meios complementares de diagnóstico não-invasivos mais completos, apenas limitada pela sua disponibilidade. Dois dos contextos clínicos em que a RMC se destaca são a suspeita de miocardite e a determinação do mecanismo fisiopatológico de lesão miocárdica em suspeitas de SCA que não apresentavam doença coronária obstrutiva. Nestes casos, além da determinação exata da fração de ejeção ventricular, fator de importância prognóstica na maioria das doenças cardíacas, a RMC permite realizar uma "avaliação histológica" não-invasiva, nomeadamente, mas não só, através da avaliação do padrão de realce tardio (RT) do miocárdico. Esta técnica permite detetar a acumulação extracelular de contraste (gadolínio) cerca de 7-10 minutos após sua administração, o que funciona particularmente bem na cardiopatia isquémica, para a qual foi inicialmente descrito, ${ }^{10}$ ao demonstrar as cicatrizes subendocárdicas de enfarte, com maior ou menor extensão transmural. A sequência de RT pode também mostrar acumulações de contraste subepicárdicas ou intramurais no caso das miocardites, ${ }^{11}$ bem como outros

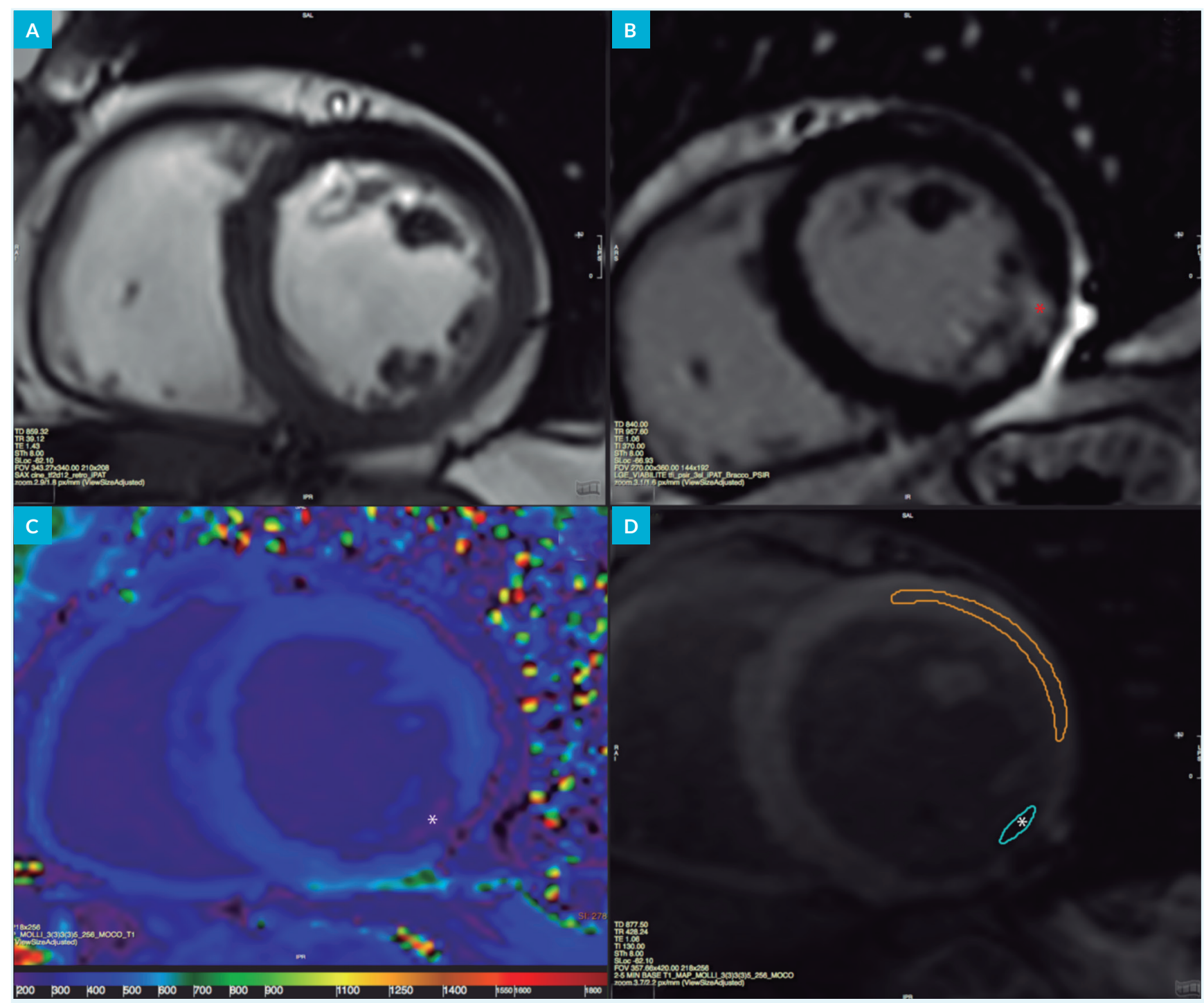

FIGURA 2. Observa-se um corte de eixo curto ventricular, com uma "cicatriz" de realce tardio subendocárdico por enfarte prévio (B), na parede ínfero-lateral do ventrículo esquerdo (asterisco). Em C observa-se a mesma região com a técnica de T1 mapping, que permite quantificar as características tecidulares da região enfartada e do miocárdio nativo (ROI laranja).

Adaptado de Teixeira T, et al. Myocardial Partition Coefficient of Gadolinium: A Pilot Study in Patients with Acute Myocarditis, Chronic Myocardial Infarction, and in Healthy Volunteers. Can J Cardiol. 2019;35:51-60.13 
padrões em miocardiopatias e doenças infiltrativas. ${ }^{12}$ Nesta avaliação histológica incluem-se ainda a deteção de edema ventricular, recentemente tornada mais robusta pela quantificação das características tecidulares miocárdicas com as técnicas de T1 e T2 mapping, e a determinação quantitativa do volume extracelular ou do coeficiente de partição (Fig. 2), ${ }^{13}$ que ultrapassam algumas limitações do RT em doenças com afeção mais difusa do miocárdio.

Todas estas características tornariam a RMC o teste de eleição para avaliar o mecanismo fisiopatológico de afeção cardíaca da COVID-19, tendo em conta as hipóteses admitidas. No entanto isso não é possível durante a fase aguda desta doença. As razões são várias, como o facto de não se conseguir na maioria das instituições dispor de dois scanners, mantendo trajetos de "limpos" e "sujos"; a dificuldade logística de doentes mais graves, muitas vezes ventilados, acederem a um scanner com ventilador compatível com ressonância, e a posterior desinfeção do mesmo; a dificuldade de doentes não-ventilados colaborarem com as apneias do exame para garantir boa qualidade de imagem; ou a longa duração do exame que aumenta a possibilidade de transmissão de infeção. De facto, as características desta infeção, e sobretudo a variância da sua gravidade que tornam o seu desfecho imprevisível, levaram várias sociedades científicas a emitir sugestões acerca dos exames complementares a utilizar, priorizando aqueles capazes de dar maior informação num menor tempo de aquisição, além da recomendação de realizar apenas os exames complementares estritamente necessários à orientação dos doentes, tudo por forma a reduzir a transmissão da infeção aos profissionais de saúde envolvidos. Essas recomendações deram prioridade à ultrassonografia POCUS, nas formas cardíaca e torácica, para o diagnóstico de complicações cardíacas e pulmonares da COVID-19.

E se já pudéssemos falar na COVID-19 no passado, satisfeitos pela forma como a sociedade portuguesa conseguiu conter relativamente a doença, dir-se-ia que a RMC seria acessória, apenas para esclarecer dum ponto de vista académico a fisiopatologia de afeção cardíaca mais frequente. Mas a realidade não será essa. Após a primeira vaga duma doença com estas características, e à medida que o desconfinamento social se iniciar, uma segunda ou terceira vagas são esperadas, até à disponibilização duma vacina ou dum tratamento eficaz. E estas vagas poderão ser mais significativas do que a primeira, sobretudo em países que conseguiram conter a dimensão desta. E por essa razão, pela necessidade de após os primeiros esforços de contenção, necessitarmos agora de perceber melhor os casos mais graves da doença para a atacar mais eficazmente no futuro, a RMC poderá ser essencial. Será necessário que os doentes que tiveram afeção cardíaca e conseguiram ultrapassar a doença, sejam orientados à realização da RMC, e avaliando, entre outros, o padrão de RT, se possa perceber o principal mecanismo fisiopatológico.

A hipótese inicial com maior racional é a de que a afeção cardíaca ocorreria através de alguma forma de miocardite linfocítica, com grau variável de disfunção ventricular. Esta não é exclusiva de idades mais jovens, mas costuma ser menos prevalente nos idosos. De facto, há relatos de miocardite fulminante por COVID-19, apresentando-se como choque cardiogénico inicial. ${ }^{14}$ No entanto, no perfil de disfunção miocárdica tardia da COVID-19, existem relatos de ausência de miocardite nas autópsias de alguns dos doentes com apresentação respiratória e evolução para síndrome de dificuldade respiratória aguda (ARDS), ${ }^{15}$ isto apesar da presença de alguns infiltrados mononucleares intersticiais no miocárdio, indiciando que a elevação de troponina nestes casos pode apenas dever-se a uma disfunção multiorgânica por síndrome de resposta inflamatória sistémica (SIRS). Por outro lado, a isquémia por mismatch necessidade-fornecimento poderá ser extremamente prevalente nestes dentes, originando enfartes tipo 2 , sendo que muitos dos doentes mais graves apresentam vários fatores de risco cardiovasculares, e estiveram sujeitos a um estado de elevada demanda energética. A RMC poderá esclarecer se algum destes mecanismos é frequente na COVID-19, pela avaliação do padrão de "cicatriz", ou não menos importante, pela relativa ausência da mesma. Se as "cicatrizes" subepicárdicas ou intramiocárdicas forem muito prevalentes, a hipótese de miocardite linfocítica deve ser considerada em doentes que se apresentem numa segunda vaga com disfunção ventricular, e apesar do diagnóstico definitivo só se poder fazer por biópsia, admitir-se um limiar mais baixo para considerar o tratamento com corticoides ou imunoglobulina endovenosa em casos extremos, tratamentos que não estão indicados para a apresentação respiratória da COVID-19. Nos casos de elevação de troponina em contexto de síndrome da resposta inflamatória sistémica (SIRS) ou em enfartes tipo 2 não se observará significativa "cicatriz", pelo que, se a ausência da mesma for a tendência nas RMC destes doentes, a elevação dos marcadores de disfunção miocárdica deve apenas ser utilizada nos doentes duma eventual segunda vaga para monitorização de prognóstico, sem qualquer terapêutica dirigida exceto a do controlo dos fatores de risco cardiovasculares. A presença de "cicatriz" permitirá ainda futuras decisões em doentes que tenham apresentado disritmia ventri- 
cular durante o internamento, sendo que a sua ausência permitirá atribuir a disritmia à provável ação iatrogénica de alguns dos fármacos empiricamente utilizados no tratamento da COVID-19 (hidroxicloroquina e azitromicina). Finalmente, eventuais "cicatrizes" de enfarte (Fig. 2) nos doentes que se escusaram a procurar o serviço de urgência, dar-nos-á uma imagem da magnitude da gravidade da pandemia, ainda que duma forma indireta, e ajudará a medicar corretamente essa franja de doentes.

Infelizmente todas estas inferências só nos serão disponíveis quando reportadas por outros países nos quais a primeira vaga da COVID-19 tenha tido outro tipo de impacto, pois não podemos esquecer que até 85\% dos doentes têm apenas doença ligeira, e nem todos os doentes internados apresentam esta afeção cardíaca, pelo que a "pegada" portuguesa provavelmente terá números reduzidos para conclusões definitivas a este nível. Ainda assim, é nossa convicção que a RMC vai ajudar a clarificar o mecanismo de afeção cardíaca desta terrível doença, e poder orientar tratamentos futuros.

\section{RESPONSABILIDADES ÉTICAS}

CONFLITOS DE INTERESSE: Os autores declaram a inexistência de conflitos de interesse na realização do presente trabalho.

FONTES DE FINANCIAMENTO: Não existiram fontes externas de financiamento para a realização deste artigo.

CONFIDENCIALIDADE DOS DADOS: Os autores declaram ter seguido os protocolos da sua instituição acerca da publicação dos dados de doentes.

CONSENTIMENTO: Consentimento do doente para publicação obtido.

PROVENIÊNCIA E REVISÃO POR PARES: Não comissionado; revisão externa por pares.

\section{ETHICAL DISCLOSURES}

CONFLICTS OF INTEREST: The authors have no conflicts of interest to declare.

FINANCING SUPPORT: This work has not received any contribution, grant or scholarship.

CONFIDENTIALITY OF DATA: The authors declare that they have followed the protocols of their work center on the publication of data from patients.

PATIENT CONSENT: Consent for publication was obtained.

PROVENANCE AND PEER REVIEW: Not commissioned; externally peer reviewed.

\section{REFERÊNCIAS}

1. Roylab Stats. [1 May 2020]. Available from: https://youtu.be/ NMre6IAAAiU (2020).

2. Walls AC, Park YJ, Tortorici MA, Wall A, McGuire AT, Veesler D. Structure, Function, and Antigenicity of the SARS-CoV-2 Spike Glycoprotein. Cell. 2020;181:281-92.e6. doi: 10.1016/j. cell.2020.02.058.

3. Siddiqi HK, Mehra MR. COVID-19 illness in native and immunosuppressed states: A clinical-therapeutic staging proposal. J Heart Lung Transplant. 2020;39:405-7. doi: 10.1016/j. healun.2020.03.012.

4. Guan, W. J. et al. Comorbidity and its impact on 1590 patients with Covid-19 in China: A Nationwide Analysis. Eur Respir J. 2020 (in press). doi: 10.1183/13993003.00547-2020.

5. Onder G, Rezza G, Brusaferro S. Case-Fatality Rate and Characteristics of Patients Dying in Relation to COVID-19 in Italy. JAMA. 2020 (in press). doi:10.1001/jama.2020.4683.

6. Dong N, Cai J, Zhou Y, Liu J, Li F. End-Stage Heart Failure With COVID-19: Strong Evidence of Myocardial Injury by 2019-nCoV. JACC Heart Fail. 2020;8:515-7. doi: 10.1016/j. jchf.2020.04.001.

7. Fried JA, Ramasubbu K, Bhatt R, Topkara VK, Clerkin KJ, Horn E, et al. The Variety of Cardiovascular Presentations of COVID-19. Circulation. 2020 (in press). doi: 10.1161/CIRCULATIONAHA.120.047164.

8. Clerkin KJ, Fried JA, Raikhelkar J, Sayer G, Griffin JM, Masoumi A, et al. COVID-19 and Cardiovascular Disease. Circulation. 2020;141:1648-55. doi:10.1161/CIRCULATIONAHA.120.046941.

9. Shi S, Qin M, Shen B, Cai Y, Liu T, Yang F, et al. Association of Cardiac Injury with Mortality in Hospitalized Patients With COVID-19 in Wuhan, China. JAMA Cardiol. 2020 (in press). doi: 10.1001/jamacardio.2020.0950.

10. Kim RJ, Shah DJ, Judd RM. How we perform delayed enhancement imaging. J Cardiovasc Magn Reson. 2003;5:505-14. doi: 10.1081/jcmr-120022267.Erratum in: J Cardiovasc Magn Reson. 2003;5:613-5.

11. Friedrich $M G$, Sechtem U, Schulz-Menger J, Holmvang G, Alakija P. Cooper LT, et al. Cardiovascular magnetic resonance in myocarditis: A JACC White Paper. J Am Coll Cardiol. 2009;53:1475-87. doi: 10.1016/j.jacc.2009.02.007.

12. Mahrholdt H, Wagner A, Judd RM, Sechtem U, Kim RJ. Delayed enhancement cardiovascular magnetic resonance assessment of non-ischaemic cardiomyopathies. Eur Heart J. 2005;26:1461-74. doi: 10.1093/eurheartj/ehi258.

13. Teixeira T, Hafyane T, Jerosch-Herold M, Marcotte F, Mongeon FP. Myocardial Partition Coefficient of Gadolinium: A Pilot Study in Patients with Acute Myocarditis, Chronic Myocardial Infarction, and in Healthy Volunteers. Can J Cardiol. 2019;35:51-60. doi: 10.1016/j.cjca.2018.10.005.

14. Hu H, Ma F, Wei X, Fang Y. Coronavirus fulminant myocarditis saved with glucocorticoid and human immunoglobulin. Eur Heart J. 2020 (in press). doi: 10.1093/eurheartj/ehaa190.

15. Xu Z, Shi L, Wang Y, Zhang J, Huang L, Zhang C, et al. Pathological findings of COVID-19 associated with acute respiratory distress syndrome. Lancet Respir Med. 2020;8:420-2. doi: 10.1016/S2213-2600(20)30076-X 